\title{
Portable Situation-Reporting System by a Palmtop Humanoid Robot for Daily Life
}

\author{
Yasumoto Ohkubo ${ }^{1}$, Kei Okada ${ }^{1}$, Takeshi Morishita ${ }^{1,2}$, Masayuki Inaba ${ }^{1}$ and Hirochika Inoue ${ }^{1}$ \\ ${ }^{1}$ Department of Mechano-Informatics, University of Tokyo \\ 7-3-1 Hongo, Bunkyo-ku, Tokyo, 113-8656 Japan \\ Email: \{ohkubo, k-okada, morisita, inaba, inoue\} @jsk.t.u-tokyo.ac.jp \\ ${ }^{2}$ Sagamidai Technical High School, Kanagawa, Japan
}

\begin{abstract}
We propose the portable situation reporting system by a small robot for daily life, which demands the rapidly system reconstitution for daily life support. In this paper, we established a system by an attachment mechanism assisted the robot system based on device-distributed approach and the patterned processing by the tree structuring of sensor information processing. The attachment mechanism facilitates the hardware system reconstitution and the patterned processing simplifies the software system restructuring. We evaluated the reconfigurable system not to re-write programs and judged the effectiveness of the system by the experiments.
\end{abstract}

\section{INTRODUCTION}

In daily life, we often get into a situation where we must watch something in a long. If robots monitor and report such situations in our place, we make things far easier. However, all robots cannot support in a wide environment such as daily life because all robots support area is limited. The purpose of this research is to design a portable situation reporting system to cover a wider workspace.

At the present day, robots monitoring life environment include Robotic Room [1] proposed by Sato et al. and robots controlling home information appliances [2] and house sitter robot developed by a business enterprise. However, because Robotic Room is room-shaped robot, Robotic Room cannot support in all living space. Also, because robots controlling home information appliances and home sitter robot can support only at home, they cannot support in all living space. But in future not only service robots [3] but also all home robots are not exclusive robots but universal robots about job family of the robots in economical aspect. Therefore, we proposed the daily life supporting system by the small robot which we can carry around every corner all of the time.

In the system, portable robots that we can carry around support us by reporting situation in daily life. We need to attach the sufficient ability for support to small robots. Realization of the system requires the design of reconfigurable ability, in which robots can change the software system and the hardware system readily depending on circumstances. The reconfigurable software system design adopts two different approaches depending on the complexity of sensor input data. The one approaches is simple information processing adaptable to a huge variety of one-dimensional sensor data. The other approach is complex information processing adaptable only to a certain multidimensional sensor data. In addition, we adopted the device-distributed approach [4] in the reconfigurable hardware system design. By robot system based on the device-distributed approach we realize the attachment mechanism, in which robots can change and add parts, for example hardware parts and sensor device, easily. We aim to support human in daily life by the mentioned system.

In the next section, we survey and analyze the related works and clarify the aim of portable situation reporting system. In Section 3, we give a summary account of the realizing method of the easily reconfigurable system. We explain the approach for realizing the reconfigurable hardware system and the developed robot based on the approach in Section 4. In Section 5, we deliver the reconfigurable software system divided into two methods depending on the complexity of sensor input data. In Section 6, we test the situation reporting system on the developed robot. In Section 7, we state the paper's conclusion and expansivily of the portable situation reporting system.

\section{Portable Situation-Reporting System}

The situation reporting support by portable robots is designed to support over all living place. To realize the support over all living place by situation reporting require constructing hardware and software for the purpose of adaptations to the all living place. Therefore, establishment of the portable situation reporting system needs the hardware system, which balance portability for carrying around and highly functionality for reporting situation, and the software system that we can restructure without programming.

\section{A. System Balancing Portability and highly functionality}

Because situation reporting behaviors need many sensors and actuators, the situation reporting behaviors by small portable robots require the robot system composition that have no limitation of the number of devices on a robot and retain the small size of the robot. The robot system is structured by device-distributed approach, and we realized the hardware system balancing portability and highly functionality by selecting the necessary devices among many devices and building the devices into the system depending on the situation.

\section{B. Reconfigurable System Without Program}

In all process of situation reporting support, the most influenced process by surrounding environments is the 
process of watching situation. Watching situation well adapted for environments variation by restructuring watching system depending on the situation enable the accommodation the all situation reporting system to a broad range of situation. To restructure the system depending on the situation requires the establishment of variable system without reprogramming and easily reconfigurable system for situation reporting. We propose the following two approaches as the realizing method of the easily reconfigurable system.

- Fixing software approach by processing a number of sensor information without changing software system

- Fixing hardware approach by processing one class of complex sensor information by changing software patterned for easily establishment of the system

\section{Portable Robot System based on Device Distributed INTELLIGENT DEVICE UNIT}

\section{A. Intelligent Device Unit}

Intelligent device units are the devices integrated with device controller, which have their own processors with communication and calculation functions. In this research, the microprocessors built in intelligent device units are Cygnal C8051F300 manufactured by CYGNAL Integrated Products, Inc. The appearance and specification of C8051F300 is listed in Fig.1. The package of C8051F300 is MLP-11 which $3 \times 3 \mathrm{~mm}$ size. The MLP-11 package has eleven-port pin, two for ground and power supply, one for reset signal, eight pins for $\mathrm{I} / \mathrm{O}$ port. Crossbar decoder mechanism maps the port pins to internal hardware peripherals on the device. Because in the eight I/O port pin of C8051F300 in the developed sensor board two pins are assigned to communicate and one pin is assigned to download the firmware of C8051F300, rest of the five pins for $\mathrm{I} / \mathrm{O}$ port is the pin for input of sensor information.

Also, every intelligent device unit has its own ID number, and the robot system distinguishes all devices by the ID number in communication between buses wired devices. Therefore, when we trade a device that another device that has the same ID number given to the device, the programs of the robot system does not need to modify.

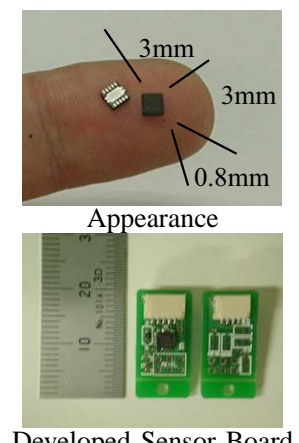

\begin{tabular}{|l|l|}
\hline CPU Core & 8051 \\
\hline Clock & $25 \mathrm{Mips} /$ Peak \\
\hline Flash Memory & $8 \mathrm{k}$ byte \\
\hline RAM & 256 byte \\
\hline Operating Voltage & $2.7-3.6 \mathrm{~V}$ \\
\hline Internal Oscillator & Yes \\
\hline I/O ports & $8(5 \mathrm{~V}$ talerant $)$ \\
\hline Uart & 1 \\
\hline $\mathrm{I}^{2} \mathrm{C} / \mathrm{SMBus}$ & Yes \\
\hline Timer & $16 \mathrm{bit} \times 3$ \\
\hline ADC & 8 bit $\times 3$ \\
\hline PCA & Yes \\
\hline Package & MLP11 $(3 \times 3 \mathrm{~mm})$ \\
\hline \multicolumn{2}{|c|}{ Specification } \\
\hline
\end{tabular}

Fig. 1. Super-micro processor : Cygnal C8051F300

\section{B. Device Distributed Approach}

The robot system based on device-distributed approach is composed of bus connected intelligent device units that each device has a processor with communication and computation function. In this system, because the number of devices the robot system can build in is not relate to the number of I/O port pins of processor, the system have no limitation of the number of devices on a robot.

In the system composition based on device distributed approach, because each processor control one device only, by introducing device distributed approach, adding a new device to a robot's body is just adding it to an existing bus network, and no significant modification of software of other device units and no significant rewiring of an onbody bus network is required. Compared to other robot systems such as on-body centralized approach [5] that one processor on a body controls all devices and module distributed approach [6] that several processors communicate each other and control several devices, device distributed approach is able to add additional devices more easily. In terms of the expandability of system, we regard the robot system based on device-distributed system as the most extensible system. Consequently, in this research, we produced the robot bus-connected developed intelligent device units based on device-distributed approach.

\section{Attachment Mechanism}

The attachment mechanism was used here for maintaining the small robot size, which is able to add additional parts that have the necessary function for the situation at the time. The additional parts are sensor device units and hardware parts. The hardware parts are the parts making preparations to monitor and bear no relation to robot system. However sensor device units as the other additional parts are intelligent device units that need to be incorporated in the system of on-body bus network in the robot, and when new parts addition to the robot system the necessity of software systemic change accrues. In general robot system, incorporating new parts in system demands serious systemic change, while little bit software change enable new parts addition in the robot system based on device distributed approach. Hence the attachment mechanism is easily able to add additional parts to the robot system for hardware parts and sensor device units, we can easily change sensor device units whose exchange is fraught with system reconfiguration as well.

Actual developed attachment parts are displayed in Fig.2. The sensor device units such as (A), (B) and (C) are adapted to connect up each analog output sensor to sensor board. The sensor device units are the parts that output sensor information getting by analog-to-digital conversion of sensor value. In the meantime, Parts of (D) is the hardware parts that fit in adsorptive cupules on the sole. The hardware parts enable us to install robots in shaky ground and these places on which the robot cannot stand statically such as ceiling and wall. 

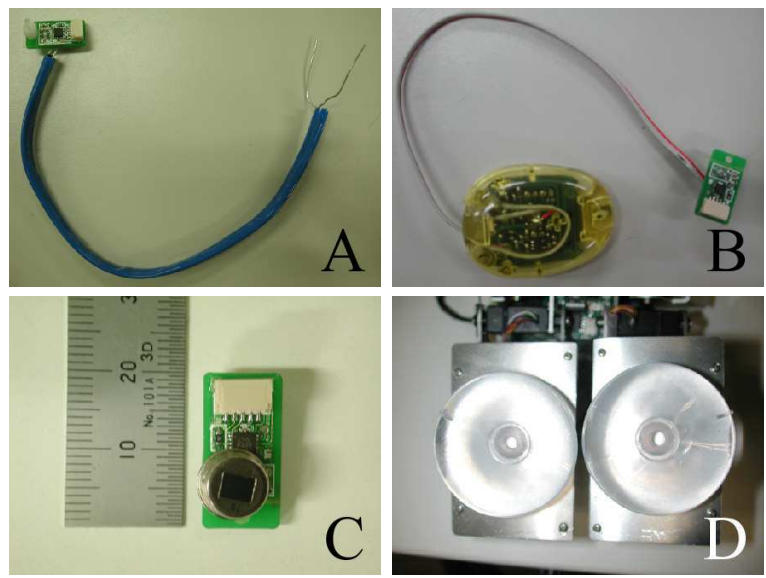

\begin{tabular}{|l|l|}
\hline Sensor Device Unit & $\begin{array}{l}\text { (A) Resistance Sensor } \\
\text { sensor for checking physical object } \\
\text { compared with reference resistance }\end{array}$ \\
\cline { 2 - 2 } & $\begin{array}{l}\text { (B) Radiowave Sensor } \\
\text { sensor for checking reception }\end{array}$ \\
\cline { 2 - 2 } & $\begin{array}{l}\text { (C) Pyroelectric Sensor } \\
\text { sensor for detecting infra-red ray } \\
\text { irradiated from human body }\end{array}$ \\
\hline Hardware Parts & $\begin{array}{l}\text { (D) Cupule foot } \\
\text { parts for standing still on the } \\
\text { hard-to-set places }\end{array}$ \\
\hline
\end{tabular}

Fig. 2. Developed Attachment Parts

\section{Small Humanoid for Situation reporting Assistance}

Hardware for situation reporting behavior demand easily installed ability and mobility for move to checking points. We selected portable humanoids as hardware of such abilities, for the portable humanoids have hands for grasping objects and legs for moving and can be carried very easily. Developed robot shown in Fig. 3 is constructed in the robot system based on device-distributed approach. Therefore, the robot can trigger the attachment mechanism. As a result, the developed robot is so small that the robot perch on its master's hand, but can report a huge variety of situations by changing function optionally.

The developed robot is produced by the remote-brained approach [7]. The robot connects the wire for Power feed and Personal Computer connection, and the vision and voice recognition systems are based on Personal Computer.

TABLE I

MAIN SPECIFICATION OF CHIBIKAZ

\begin{tabular}{|l|l|}
\hline DOF & $18($ Head:2 Arms: $4 \times 2$ Legs: $4 \times 2)$ \\
Size & $220 \times 160 \times 100[\mathrm{~mm}]$ \\
Weight & $750[\mathrm{~g}]$ \\
Motor Unit & \\
Servo Motor & S3102(Futaba product) \\
Built-in Processor & C8051F300(Cygnal product) \\
Camera & $1 / 3$ inch CMOS Image Sensor \\
Acceleration Sensor & ADXL202(Analog Devices product) \\
Control Voltage & $6[\mathrm{~V}]$ \\
Motor Voltage & $6[\mathrm{~V}]$ \\
\hline
\end{tabular}

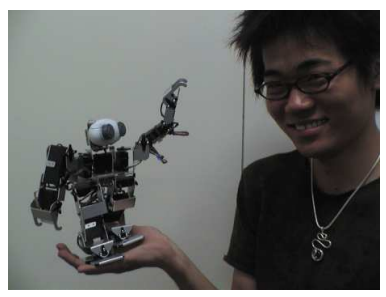

Appearance of ChibiKaz

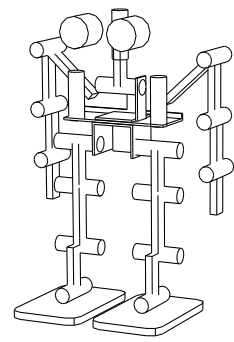

DOF design
Fig. 3. Palm-Top Humanoid:ChibiKaz

\section{Sensor Information Processing System}

The situation reporting system illustrated in Fig.4 is broken into three phases. The first phase is the phase in which the robot receives the direct human command, by which the human selects the behavior pattern. In the second phase the robot gets ready for watching situations by reconfigure the necessary software system to sensor information processing and detects the change of the situations. In last phase, the robot communicates the information that the robot got in second phase.

The first phase and the last phase are the phases communicating with human, smooth communication with human are important. In this research, as means of smooth communication we selected interlocution by speech understanding, sending and receiving emails by accessing the mail server and movement by motility of the robot. The robot decides the watching system by human instructions obtained from these means, and reconfigures the situational watching software system.

Sensors are classified into simple sensors outputting only single-species information such as pressure sensor and complex sensors outputting wide variety of information such as image sensor outputting visual information. Sensor information processing system can change processing software for class of input information. In fact, software system for simple sensors is equal to software system for complex sensors in information processing.

\section{A. Simple sensor data processing}

Because Simple sensors output only one-dimensional data, class of information obtained from the sensors is largely similar to class of the sensors. Robots cannot get information except limited data outputting from simple sensors that the robots can attach. The robots hardly can match a huge variety of situations. The robots with attachment mechanism adapt to a huge variety of aspects by the situational select-and-change sensor device units.

However, if each and every sensors demand processing software, the robot system needs to change the software severely when the change of sensor device units and it is impossible to deal quickly with each situation. The realization of speedy reconfiguration by simplification of the system requires many sensors processible software system. Consequently, when the robot uses the simple 
sensors, by use of one software for different sensors and change of sensors as if unfolds, we established the easily reconfigurable system to respond to a variety of situations.

Actually, if we attend to the change of sensors data, how sensors data change fall into two types. One is the extraordinary changing type in which sensor value change significantly when situations change such as pyroelectric sensors and radiowave sensors. The other is the gradually change type in which the sensor value always change little by little such as temperature sensors and humidity sensors. Because the patterns of sensors divided into two classes, information processing software requires only two patterns associated with the two classes of sensors change. As examples of the change pattern of the former the changes of pyroelectric sensor value and radiowave sensor value are listed in Fig.5 and Fig.6. It is a fact that the changes of sensor value are abrupt. In consequence, the software for change pattern of the former is to detect the drastic change of the present sensor values by setting the right threshold. Also, the software for the latter change pattern is to detect the change of sensor value to specific values by setting the values as threshold. The two software systems are about equally apart from setting threshold. Therefore, we can configure the system which can switch the software for the extraordinary change type and the gradually change type by whether one sets threshold.

\section{B. Complex sensor data processing}

When the robot use the complex sensors outputting multi-dimensional data, the robot can get a huge variety of information from one sensor by change of the feature extraction methods. One sensor can respond to a large variety of situations, for one sensor can play the role of many sensors by change of the feature extraction methods. In other words, we can create the easily configurable system capable of responding appropriately to a multiple of situations by the change of not sensors but software system depending on circumstances.

The rapid reconfigurability is important for the change of software on site. Because the robot can select the adequate software by external command input, the rapid reconfiguration is realized. The hierarchy structure of software shown in Fig.7 enable us to select the best processing methods or the best processing parameters in all ranks. When we make the hierarchy structure, we make the first rank the rank selecting sensors and make the second rank the rank selecting sensor data processing methods. All the rank after the third rank becomes the rank selecting processing parameters. The upper parameters are, the important the parameters are for sensor data processing.

In example of Fig.7, as the methods of inputting data processing from camera of image sensor we choose correlation [8] from a variety of processing methods such as color extraction and edge detection. Moreover, as screen split mean necessary for correlation we select simple screen tripartition. In practice the robot can construct software system on watching site rapidly by setting connection between all parameters in all ranks and input information.

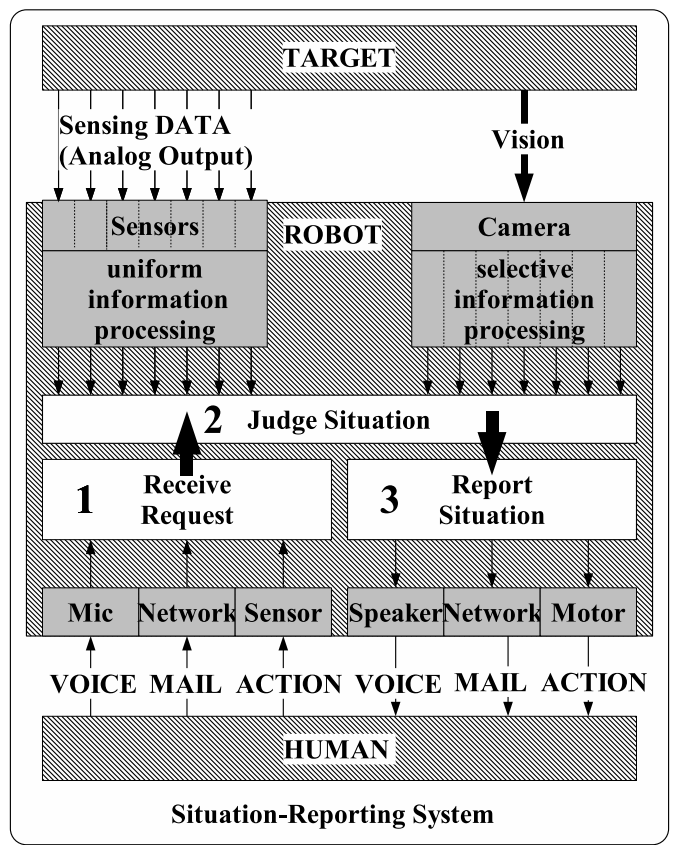

Fig. 4. Situation reporting system

First step is the step for receiving human requests and the robot behavior is determined by the human request. Second step is the step for judging situations and the robot checks the situation based on the behavior decided in the first step. Last step is the step for reporting situations and the robot reports the information got in the second step.

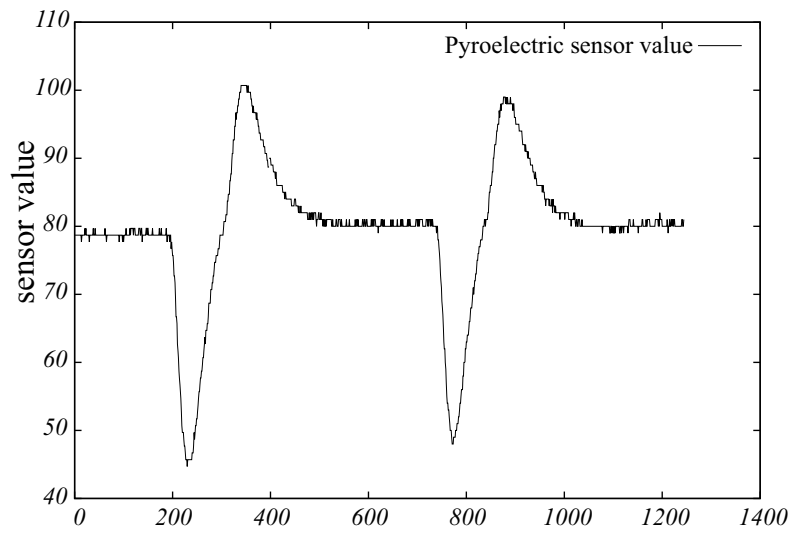

Fig. 5. The change of pyroelectric sensor value

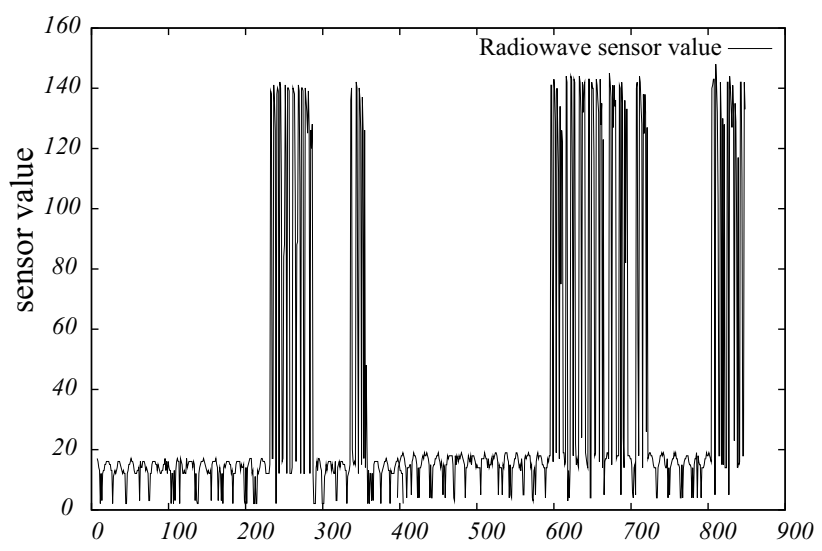

Fig. 6. The change of radiowave sensor values 

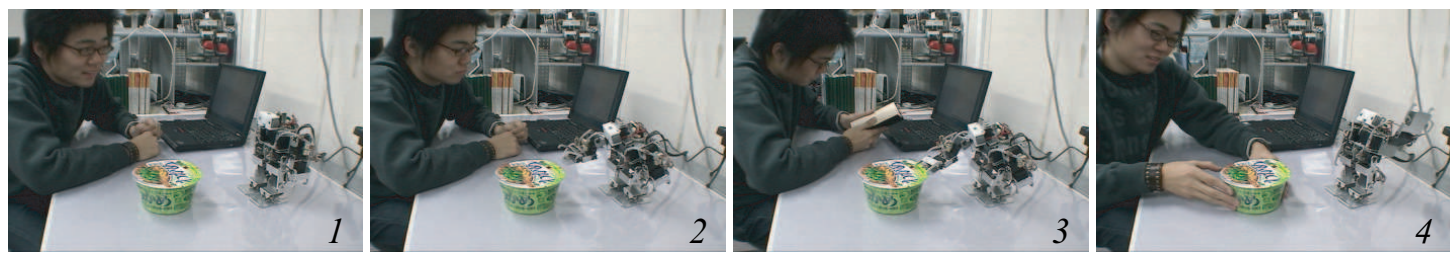

Fig. 8. (A) When a man asks the robot to time cooking cup noodle, the robot grabs the cup and unclench the hands after three minutes.
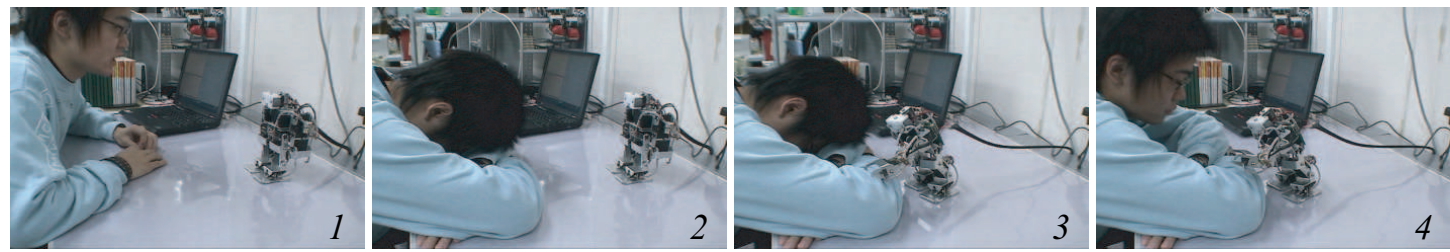

Fig. 9. (B) When a man asks the robot to wake from sleep after one hour, the robot wakes him from sleep by approaching and touching him in fact.

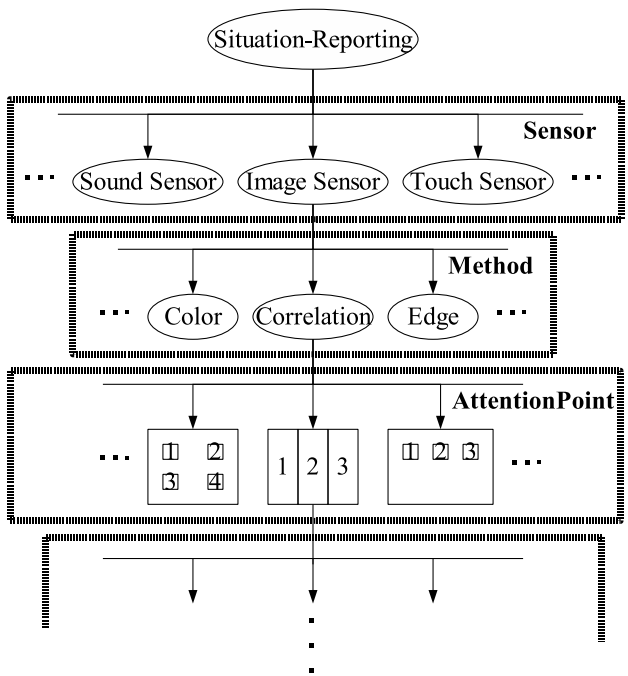

Fig. 7. Hierarchization of setting of processing parameters

\section{EXPERIMENT FOR INSPECTING SITUATION-REPORTING SYSTEM}

The robot completion carrier ((A) Fig.8) and the robot timekeeper ((B) Fig.9) are the change detection experiments of the gradually changing type in information processing of simple sensors. In both (A) and (B) we instructs the behavior of the robot by voice. Though (A) and (B) are these situation reporting behaviors based on the same assessment measure of the situation, (A) and (B) are the different behaviors. The system architectonics which need no rewriting programs in setting of these behaviors requires to set threshold and behaviors by external input.

The detection of open and close ((C) Fig.10) and the measure of the water in pot ((D) Fig.11) is the examples of the situation reporting behaviors classified into the change detection of the extraordinary changing type. (C) and (D) indicate that the same programs are adaptable to different sensors such as acceleration sensors and resistance sensors. This like kind of programs constitutes the system, which do not need to rewrite programs in the change of sensors based on attachment mechanism.

The visiting report ((E) Fig.12) and the watch passage ((F) Fig.13) fall into the category of sensor data processing. Processing in both (E) and (F) is based on correlation processing, and the robot extract color shading information in specific locations of the robot vision by correlation processing. The robot carries on situation reporting behaviors by finding human passage by the change of shading information. Moreover, in $(\mathrm{F})$ processing includes color extraction in addition to correlation processing, the processing software responds to more complex situations. Easily setting of complex processing software such as software used in $(E)$ and $(F)$ requires correlation parameters with external input by hierarchization of setting of processing parameters.

\section{CONCLUSION}

In this paper, we proposed the portable situation reporting system by the small humanoid that we can carry anywhere and anytime. In the system, the robot checks human life circumstances and reports the necessary event in information getting by watching circumstances. In suggested situation reporting system, we introduced the method of system reconfiguration depending on situations to match a huge variety of all situations for daily life. We constitute easily reconfigurable system by switching sensor data processing method depending to sensor data complexity.

In simple sensor data processing, attachment mechanism realized a system with no change in software even if in changing sensors. In complex sensor data processing, hierarchization of setting of processing parameters enable the software system of complex sensor data processing to reconfigure rapidly by external input. Consequently, we established the system that we can reconfigure rapidly depending on situations.

The sensor data processing system in this paper can be applied to other robots. By the system robots can change 

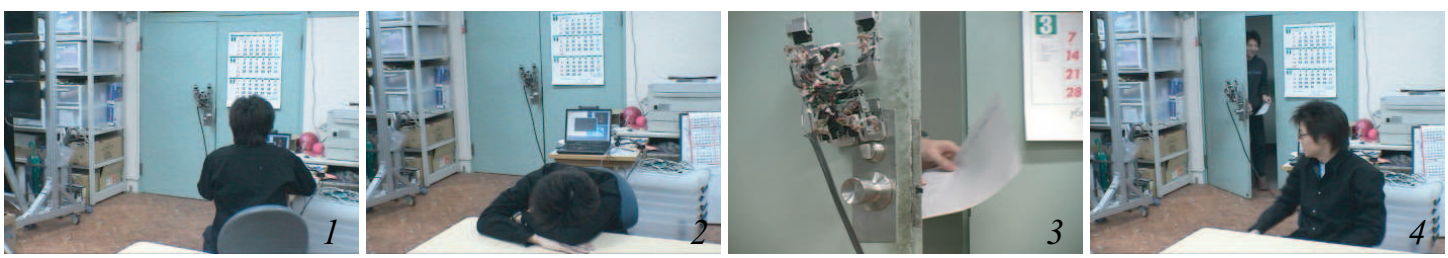

Fig. 10. (C) The robot affixing itself to the door detects opening the door by acceleration sensor data. By the behavior a man notice that someone visits in the action.
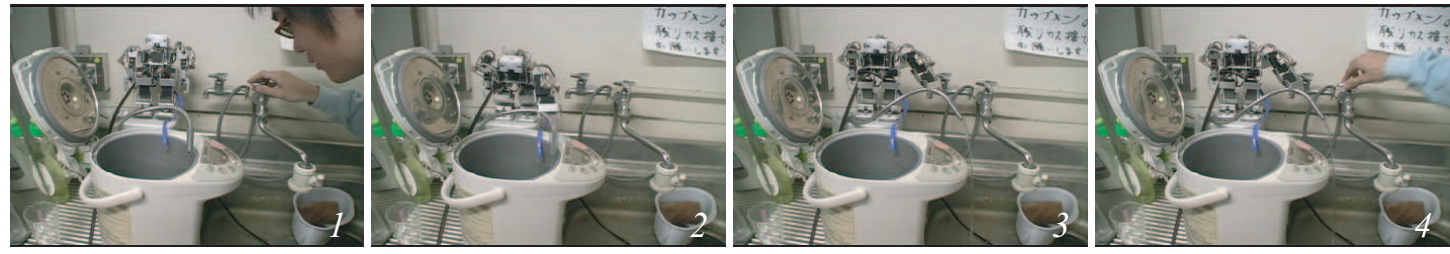

Fig. 11. (D) The robot on the pint pot gets tap-dripping water out of the way for preventing water from overflowing when water stage becomes a certain level.
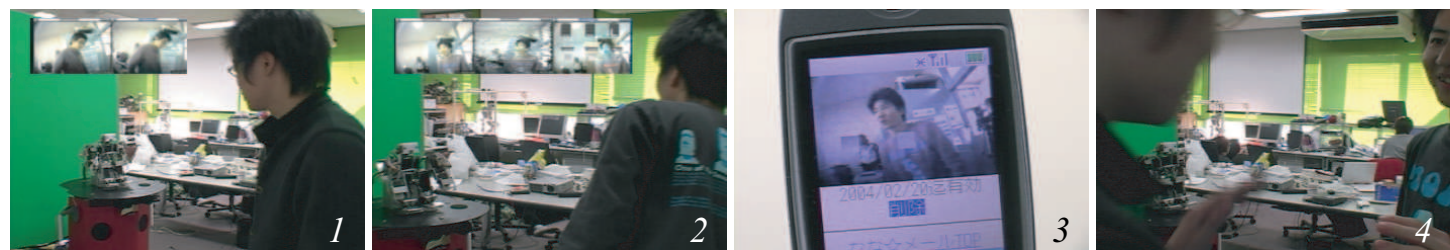

Fig. 12. (E) When a man asks the robot to housesit, at first the robot commits the vision at the time to memory. Then the robot always compares the memorized vision to the present vision and e-mails him when the present vision differs from the memorized vision greatly.
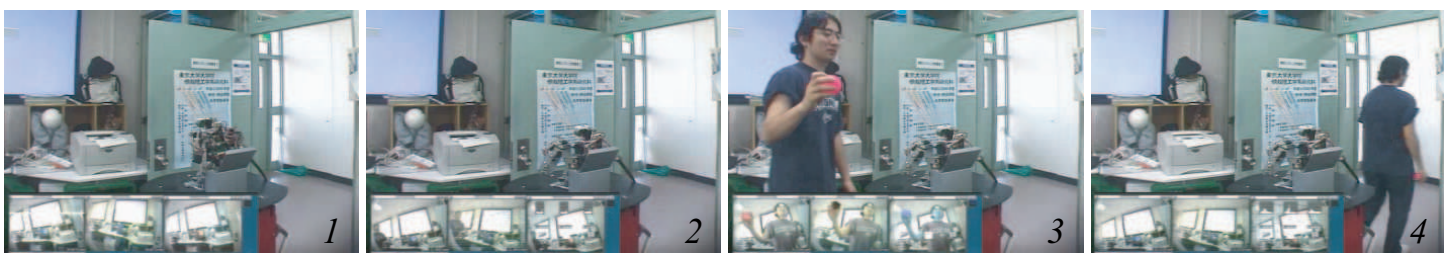

Fig. 13. (F) The robot checks comings and goings of people and the robot reports the adit. However, when a man shows the robot particular color, the robot does not report the adit. In other words, we can change the reaction of the robot by conditions such as whether especial sign is not.

sensors readily and get different information depending on conditions. In other words, robots can stretch themselves and work in a wider range of fields for human than ever.

The problem of the system is necessity for the instructions from human. The portable robots in this paper cannot decide the behavior autonomously. If robots can reconfigure the robot system autonomously depending on conditions, robots would become more adaptable robots to many different environments.

\section{REFERENCES}

[1] T. Sato, Y. Nishida, and H. Mizoguchi, "Robotic Room:Symbiosis with human through behavior media," Robotic and Autonomous Systems, vol. 18, pp. 185-194, 1996.

[2] D. Yamamoto, T. Yoshimi, J. Hirokawa, H. Nakamoto, H. Ogawa, and N. Matsuhira, "A Robotic Information Home Appliance Concept Model, "ApriAlpha"-Overview of Implemented Functions and Locomotion Control Method-," in Proceedings of the 21th Annual Conference of RSJ, 2003, p. 1E27, (in Japanese).
[3] M. Kasaka, "Service Robot develops from Exclusive to Universal Proposal for New Human-Robot Relatios," in Proceedings of the 21th Annual Conference of RSJ, 2003, p. 3H12, (in Japanese).

[4] K. Okada, A. Fuyuno, T. Morishita, T. Ogura, Y. Ohkubo, Y. Kino, M. Inaba, and H. Inoue, "Device distributed approach to extensible robot system using intelligent device unit with super-micro processor," in Journal of Robotics and Mechatronics, 2004, (to appear).

[5] H. Inoue, S. Tachi, K. Tanie, K. Yokoi, S. Hirai, H. Hirukawa, K. Hirai, S. Nakayama, K. Sawada, T. Nishiyama, O. Miki, T. Itoko, H. Inaba, and M. Sudo, "Hrp: Humanoid robotics project of miti," in Proceedings of the First IEEE-RAS International Conference on Humanoid Robots(Humanoids 2000), September 2000.

[6] N. Yamasaki, "Design and implementation of responsive processor for parallel/distributed control and its development environments," vol. 13, pp. 125-133, September 2001.

[7] M. Inaba, "Remote-brained humanoid project," in Advanced Robotics, 1997, pp. 11(6):605-620.

[8] H. Inoue, M. Inaba, T. Mori, and T. Tachikawa, "Real-Time Vision System Based on Correlation Processing," The Journal of the Robotics Society of Japan(JRSJ), vol. 13, no. 1, pp. 134-140, 1995, (in japanese). 